\title{
Avaliação da remoção de matéria orgânica carbonácea e nitrogenada de águas residuárias em biorreator de membranas
}

\author{
Evaluation of carbonaceous organic matter and nitrogen \\ removal of wastewater in membrane bioreactor
}

\author{
Luiz Fernando Bezerra \\ Mestre em Engenharia Civil pela Faculdade de Engenharia de Ilha Solteira da Universidade Estadual Paulista "Júlio de Mesquita Filho" (FEIS/Unesp). \\ Consultor Técnico da Nalco Brasil Ltda. \\ Tsunao Matsumoto \\ Doutor em Hidráulica e Saneamento pela Escola de Engenharia de São Carlos da Universidade de São Paulo (EESC/USP). \\ Professor Adjunto do Departamento de Engenharia Civil da FEIS/Unesp
}

\section{Resumo}

O presente trabalho objetivou a avaliação da remoção de matéria orgânica carbonácea e nitrogenada, bem como a determinação do fluxo crítico, em biorreator de membranas, com zona pré-anóxica, tratando águas residuárias industriais da produção de aminoácidos. O reator foi operado sob carga orgânica volumétrica de $1,91 \mathrm{~kg} \cdot \mathrm{DQO} \cdot \mathrm{m}^{-3} \cdot \mathrm{d}^{-1}$ e $0,18 \mathrm{~kg} \cdot \mathrm{NTK} \cdot \mathrm{m}^{-3} \cdot \mathrm{d}^{-1}$; a recirculação do reator aeróbio para o reator anóxico foi de quatro vezes a vazão afluente. O reator apresentou médias de remoção de DQO, NTK e NT de 97, 98 e 92\%, respectivamente. O sistema de ultrafiltração foi testado em vários fluxos entre 25 e 37 L.m².'. ${ }^{-1}$ e determinou-se o fluxo crítico de $28 \mathrm{~L} . \mathrm{m}^{-2} . \mathrm{h}^{-1}$ quando operado com 11,4 g. $\mathrm{L}^{-1}$ de SST e 35 dias de tempo de retenção celular. Os resultados mostraram que houve viabilidade técnica no uso de biorreator de membranas para remoção de matéria orgânica de águas residuárias industriais da produção de aminoácidos.

Palavras-chave: biorreator de membranas; ultrafiltração; fluxo crítico; remoção de nitrogênio.

\section{Abstract}

This study aimed to evaluate the carbonaceous organic matter and nitrogen removal as well as the determination of critical flux in membrane bioreactor, with pre-anoxic zone, treating industrial wastewater of amino acids production. The reactor was operated under organic loading rate of $1.91 \mathrm{kgDQO}^{-3} \mathrm{~m}^{3}$.day ${ }^{-1} \mathrm{and}$ $0.18 \mathrm{kgNTK}^{-\mathrm{m}^{-3}}$. day ${ }^{-1}$, the recirculation from aerobic reactor to anoxic reactor was four times the influent flow rate. The system showed an average removal of COD, TKN and TN of 97,98 and 92\%, respectively. The ultrafiltration system was tested at various fluxes between 25 and $37 \mathrm{~L} \cdot \mathrm{m}^{-2} \cdot \mathrm{h}^{-1}$, to determine the critical flux of $28 \mathrm{~L} . \mathrm{m}^{-2} . \mathrm{h}^{-1}$ operating at $11.4 \mathrm{~g} \cdot \mathrm{L}^{-1}$ of TSS and 35 days of sludge retention time. The results showed the technical feasibility of using membrane bioreactor to remove organic matter from industrial wastewater of amino acids production.

Keywords: membrane bioreactor; ultrafiltration; critical flux; nitrogen removal.

Endereço para correspondência: Luiz Fernando Bezerra - Departamento de Engenharia Civil da Faculdade de Engenharia de Ilha Solteira da Universidade Estadual Paulista "Júlio de Mesquita Filho" - Alameda Bahia, 550 - 15385-000 - Ilha Solteira (SP), Brasil - Tel./Fax: (18) 3743-1125 - E-mail: luiz_bezerra81@hotmail.com Recebido: 15/03/10 - Aceito: 11/04/11 - Reg. ABES: 04410 


\section{Introdução}

A remoção de matéria carbonácea em águas residuárias continua sendo uma exigência, mas o controle no descarte de nutrientes para o meio ambiente tem ganhado importância nessa última década no Brasil. Novas tecnologias estão sendo desenvolvidas para alcançar os requisitos cada vez mais restritivos para o descarte de nutrientes (nitrogênio e fósforo) e micropoluentes que causam impactos negativos, como a eutrofização e a deterioração da qualidade de água nos corpos receptores (CAGATAYHAN, 2008).

Especificamente para o caso de sistemas de lodo ativado, uma série de variações sistêmicas foi desenvolvida com vistas à remoção biológica de material nitrogenado que, com a introdução de zonas anóxicas, promove a redução do N-NO3-, produzido aerobiamente, como aceptor de elétrons para a estabilização da matéria orgânica, no lugar do oxigênio molecular (WEF, 2005).

Os processos biológicos de remoção de nutrientes aplicam várias combinações entre sistemas anaeróbios, anóxicos e aeróbios para remover nutriente, e por isso requerem grandes áreas para implantação. Alguns desses processos incluem os sistemas $\mathrm{A}^{2} \mathrm{O}$ (do inglês Anaerobicl Anoxic/Oxic - Anaeróbio/Anóxico/Aeróbio) e o Bardenpho de quatro estágios (Anóxico/Aeróbio/Anóxico 2/Re-aeração) (METCALF \& EDDY, 2003).

O biorreator de membranas (MBR), que combina o tradicional processo de lodo ativado com filtração por membranas, tem alta eficiência de separação líquido-sólido e produz efluente tratado de alta qualidade no tratamento de águas residuárias. Vantagens adicionais incluem a baixa área de ocupação, a resistência robusta às variações de carga do afluente, a reduzida produção de lodo e a possibilidade de projeto modular (MENG et al., 2009).

Entretanto, os custos de implantação e operação do MBR constituem um dos mais críticos obstáculos para disseminação dessa tecnologia. A maior parte dos custos operacionais se relaciona à alta demanda energética para aeração do sistema de filtração e do reator aeróbio que opera com altas concentrações de biomassa (NYWENING E ZHOU, 2009).

Ersu et. al. (2008) estudaram a remoção de material carbonáceo e nitrogenado tratando esgoto doméstico sintético contendo demanda química de oxigênio (DQO) média de $510 \mathrm{mg} . \mathrm{L}^{-1}$, 42,6 mg.L-1 de nitrogênio total (NT) e 11,4 mg. $\mathrm{L}^{-1}$ de fósforo total (PT) em um MBR submerso de placa plana em escala de laboratório composto de dois compartimentos: um anóxico e um aeróbio, onde estavam as membranas. O melhor resultado se deu com recirculação de 100\% de lodo e $100 \%$ de permeado, em relação à vazão afluente, apresentando resultados de 93,2, 76,3 e 58,7\% de remoção de DQO, NT e PT, respectivamente. O reator operou com relação alimento por microorganismo (A/M) de 0,11 a 0,22 kg DQO.kgSSV $1 . d^{-1}$ e carga orgânica volumétrica (COV) de 0,46 a 0,91 kg DQO.m-3. $\mathrm{d}^{-1}$. A relação alimento por micro-organismo relaciona a quantidade de matéria orgânica disponível como alimento para o consórcio de micro-organismos contido nos reatores.

Zoh e Strenstrom (2002) propuseram um MBR externo com membranas cerâmicas em escala de bancada tratando água residuária sintética da indústria de explosivos, contendo DQO de $357 \mathrm{mg} \cdot \mathrm{L}^{-1} \mathrm{e}$ 174 mg. L $^{-1}$ de NT, nas formas de N-NO2- e N-NO3; operando apenas com um reator anóxico (1,2L) atingiu 97\% de remoção de DQO e $66 \%$ de remoção de NT. O reator operou com 1,82 kg DQO.kgSSV ${ }^{1} \cdot \mathrm{d}^{-1}$ de relação A/M e 0,50 kgNT.kgSSV . $^{-1}$ de relação N/M. A COV máxima foi de $0,72 \mathrm{kgDQO} \cdot \mathrm{m}^{-3} \cdot \mathrm{d}^{-1}$.

Em outro trabalho, Chen et al. (2003) estudaram a remoção de DQO e nitrogênio de água residuária de indústria EPTO (electronics and photonics technology office) em um sistema MBR. A planta piloto consistia em um sistema de lodo ativado pré-anóxico com a etapa de separação sólido/líquido sendo promovida por um sistema de ultrafiltração submersa. As eficiências alcançadas na remoção de DQO, nitrogênio total Kjeldahl (NTK) e NT foram de 94, 90 e 75\%, respectivamente, com tempo de retenção hidráulica de 1,7 dia e razão de recirculação de nitratos igual a 3. A concentração de SST no tanque de aeração foi de 8 g.L $L^{-1}$ em média.

O bom funcionamento do MBR não se resume apenas ao controle do reator biológico, mas de igual importância é a operação do sistema de membranas dentro de condições específicas, que vão determinar altas produções de permeado com a qualidade desejada com o mínimo de limpezas químicas. O conhecimento do fluxo crítico é condição indispensável para se demonstrar a viabilidade técnica da aplicabilidade do MBR no tratamento de uma determinada água residuária

Segundo Bachim et al. (2006), o conceito de fluxo crítico pode ser definido como o primeiro fluxo de permeado no qual a deposição (fouling) irreversível aparece na superfície da membrana.

A medida experimental do fluxo crítico pode ser realizada impondo-se um fluxo fixo no sistema de filtração e medindo-se a pressão transmembrana (TMP) ou pela imposição de uma TMP fixa acompanhada da medição na queda do fluxo. Porém, devido às características de forte tendência de deposição que a suspensão de lodo biológico possui, segundo Judd (2006), o melhor método para essa investigação é manter um determinado fluxo e se monitorar a variação da TMP ao longo do tempo.

Guglielmi et al. (2007), tratando esgoto municipal em MBR submerso e testando fluxos que variavam de 17 a $30 \mathrm{~L} \cdot \mathrm{m}^{-2} \cdot \mathrm{h}^{-1}$, em ensaios de longo prazo de operação, encontraram fluxo crítico de 28 $\mathrm{L} \cdot \mathrm{m}^{-2} \cdot \mathrm{h}^{-1}$. O reator foi operado com concentrações de sólidos de 7,7 g. $\mathrm{L}^{-1}$ com tempo de retenção celular de 20 dias.

Le Clech et al. (2006), tratando esgoto sanitário em um MBR de fibras ocas submerso, encontraram fluxos críticos de 10 e 18 L.m ${ }^{2} \cdot h^{-1}$, respectivamente, para o esgoto real e sintético. O reator operou com concentração de SST no tanque de aeração de 3 g.L.-1 para ambas as alimentações. Não houve descarte de lodo durante os ensaios. 
Yuan et al. (2010) encontraram fluxo crítico de $30 \mathrm{~L} \cdot \mathrm{m}^{-2} \cdot \mathrm{h}^{-1}$ quanto operaram MBR submerso com membranas de fibraca oca tratando esgoto sintético. O material das membrnas foi fribra de vidro com diâmetro de poro de $1 \mathrm{~mm}$. O reator operou com concentrações de sólidos de 8 g.L L $^{-1}$.

$\mathrm{Na}$ presente pesquisa, objetivou-se avaliar o desempenho do MBR, quanto aos fluxos de filtração, para o tratamento de água residuária industrial da fabricação de aminoácidos visando à remoção de matéria orgânica carbonácea e nitrogenada. Para atingir o objetivo geral proposto, estabeleceram-se os seguintes objetivos específicos: avaliação do desempenho do sistema de membranas de ultrafiltração, determinação do fluxo crítico e avaliação do tratamento biológico quanto à remoção de matéria orgânica carbonácea e nitrogenada.

\section{Material e métodos}

O presente estudo foi conduzido em um sistema piloto de MBR externas, com duas câmaras (uma anóxica e outra aeróbia), para a avaliação da remoção de matéria orgânica carbonácea e nitrogenada tratando água residuária industrial de uma fábrica de aminoácidos no interior do Estado de São Paulo.

O protótipo piloto foi operado por 63 dias, entre 15 de julho a 15 de setembro de 2009, e o estudo foi dividido em duas etapas: a primeira de testes preliminares e a segunda de operação normal, na qual foram avaliados os fluxos e as remoções do sistema biológico. A configuração do protótipo piloto de MBR é apresentada na Figura 1.
Neste artigo não são apresentados os dados da fase preliminar por se tratar de um período de ajustes.

O protótipo piloto, como mostrado na Figura 1, era composto de:

1. um pré-filtro, tipo cesto, de $500 \mu \mathrm{m}$;

2. um tanque de equalização de $1.000 \mathrm{~L}$;

3. uma bomba helicoidal de alimentação do sistema biológico de 200 L.h $^{-1}$.

4. um tanque anóxico de 600 L com agitador tipo turbina de três pás com potência de $745 \mathrm{~W}$ (1 cv);

5. um tanque aeróbio com $2.000 \mathrm{~L}$;

6. uma bomba helicoidal de recirculação de nitratos de $1.000 \mathrm{~L}^{-\mathrm{h}^{-1}}$,

7. um sistema de aeração composto por um soprador tipo roots, trilobular, com potência de $2.982 \mathrm{~W}$ (4 cv), com vazão máxima de $120 \mathrm{Nm}^{3} \cdot \mathrm{h}^{-1}$, e sistema de distribuição (instado no fundo do tanque aeróbio) composto por dez difusores de membrana tubulares de $85 \mathrm{~mm}$ de diâmetro por $1.000 \mathrm{~mm}$ de comprimento;

8. um pré-filtro, tipo cesto, de $500 \mu \mathrm{m}$;

9. uma bomba centrífuga de circulação do sistema de ultrafiltração de $16 \mathrm{~m}^{3} \cdot \mathrm{h}^{-1}$;

10. um sistema de ultrafiltração com dois módulos de membranas tubulares de 3" e $1.000 \mathrm{~mm}$ de comprimento. As fibras tubulares de poliéter sulfona (PES) tinham 5,2 mm de diâmetro e poros com diâmetros de 30 nm;

11. uma bomba helicoidal para retirada de permeado de 200 L.h.'

12. um tanque de permeado de $1.000 \mathrm{~L}$;

13. uma bomba centrífuga para retrolavagem de $1.100 \mathrm{~L} \cdot \mathrm{h}^{-1}$;

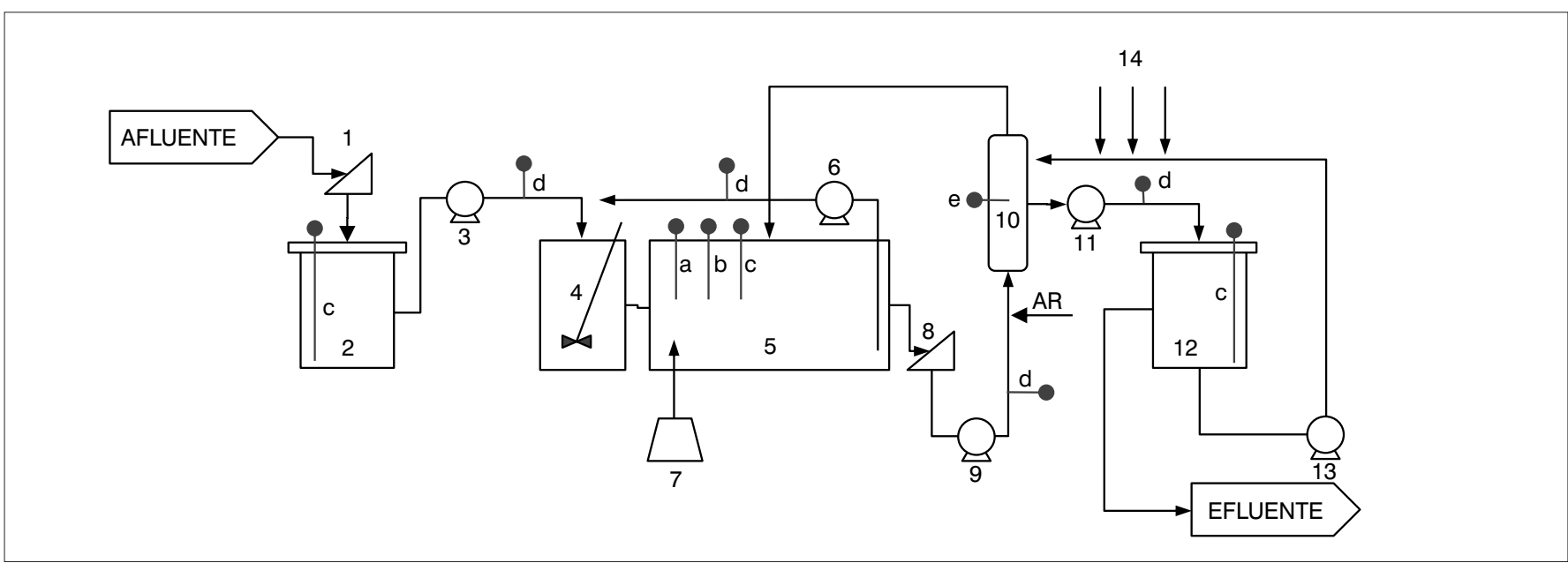

Figura 1 - Fluxograma do protótipo piloto de biorreator de membranas. 1: pré-filtro, tipo cesto, de $500 \mu \mathrm{m}$; 2: tanque de equalização de 1.000 L; 3: bomba

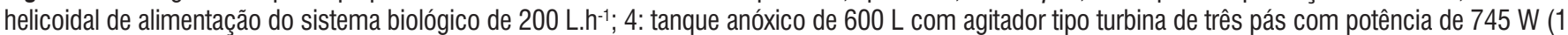
cv); 5: tanque aeróbio com 2.000 L; 6: bomba helicoidal de recirculação de nitratos de 1.000 L.h $^{-1} ; 7$ : sistema de aeração composto por um soprador tipo roots; 8: pré-filtro, tipo cesto, de $500 \mu \mathrm{m}$; 9: bomba centrífuga de circulação do sistema de ultrafiltração de $16 \mathrm{~m}^{3} . \mathrm{h}^{-1} ; 10$ : sistema de ultrafiltração com dois módulos de membranas tubulares de 3" e 1.000 mm de comprimento; 11: bomba helicoidal para retirada de permeado de $200{\mathrm{~L} . \mathrm{h}^{-1} ;}^{-12:}$ tanque de

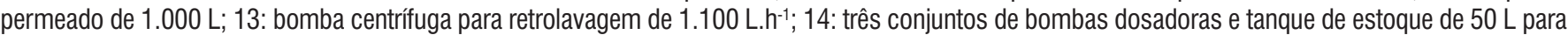
dosagem de hidróxido de sódio, ácido cítrico e hipoclorito de sódio com capacidade de 2, 5 e 20 L.h $\mathrm{h}^{-1}$, respectivamente; (a) sensor de pH; (b) sensor de oxigênio dissolvido; (c) sensores de nível; (d) medidores de vazão eletromagnéticos; (e) medidor de pressão diferencial. 
14. três conjuntos de bombas dosadoras e tanque de estoque de $50 \mathrm{~L}$ para dosagem de hidróxido de sódio, ácido cítrico e hipoclorito de sódio com capacidade de 2, 5 e 20 L.h-1, respectivamente;

15. um conjunto de instrumentação composto por um sensor de $\mathrm{pH}$ (a), um sensor de oxigênio dissolvido (b), três sensores de nível (c), quatro medidores de vazão eletromagnéticos (d) e um medidor de pressão diferencial (e);

16. uma unidade de Controle Lógico Programável (CLP) Unitronics VR350-35-R34.

O protótipo piloto de MBR foi projetado para tratar água residuária da fabricação de aminoácidos a uma vazão de 85 a 170 L.h $^{-1}$. A partir do tanque de equalização, o afluente bruto era transferido para o tanque anóxico. A vazão de alimentação era controlada por meio de um medidor eletromagnético que ajustava automaticamente (pelo CLP) a rotação do motor da bomba de alimentação

Após o tanque anóxico, a mistura era direcionada para o tanque aeróbio. Foram instalados, no tanque aeróbio, um sistema de distribuição de ar de bolhas finas, um sensor de pH e um sensor para medição do oxigênio dissolvido, ambos os sensores conectados ao CLP.

Do tanque de aeração, parte do efluente era circulado para o tanque anóxico a uma razão de uma a cinco vezes a vazão de alimentação para promover a desnitrificação.

Após o tanque de aeração o lodo circulava pela unidade de filtração, composta por dois módulos de membranas de ultrafiltração, na qual o lodo era separado do efluente tratado. O sistema de ultrafiltração era operado no modo de filtração tangencial de baixa pressão com inserção de ar durante a filtração (sistema air-lift). Durante cinco minutos, o sistema operava filtrando o lodo e, na sequência, realizava uma retrolavagem de dez segundos. Esse ciclo se repetia sucessivamente até a parada da unidade para a limpeza química. O efluente permeado produzido pelas membranas ficava estocado num tanque para ser usado na retrolavagem

As medições dos parâmetros físico-químicos seguiram as recomendações do Standards Methods for the Examination of Water and Wastewater (APHA et al, , 2005).

\section{Operação do tratamento biológico}

O sistema de tratamento biológico era controlado diariamente quanto à carga aplicada (carbonácea e nitrogenada), quanto à idade do lodo e quanto à carga volumétrica, bem como eram monitorados os parâmetros que determinavam a saúde da biomassa e o grau de eficiência na remoção dos poluentes alcançado pelo sistema.

\section{Cálculo da carga volumétrica}

A carga volumétrica é obtida dividindo-se a carga aplicada pelo volume do reator biológico, conforme Equações 1 e 2 .

$$
\begin{aligned}
& \mathrm{COV}=\frac{\mathrm{C}_{\mathrm{DQO}}}{\mathrm{V}} \\
& \mathrm{CVN}=\frac{\mathrm{C}_{\mathrm{NTK}}}{\mathrm{V}}
\end{aligned}
$$$$
\text { Equação } 1
$$

Equação 2

Onde:

COV: carga orgânica volumétrica (kg DQO. $\left.\mathrm{m}^{3} \cdot \mathrm{dia}^{-1}\right)$;

CVN: carga volumétrica nitrogenada (kg NTK.m $\left.{ }^{3} \cdot \operatorname{dia}^{-1}\right)$;

$\mathrm{C}_{\mathrm{DQO}}$ : carga aplicada (kg DQO.dia $\left.{ }^{-1}\right)$;

$\mathrm{C}_{\text {NTK}}$ : carga aplicada (kg NTK.dia $\left.{ }^{-1}\right)$;

$\mathrm{V}$ : volume do reator biológico $\left(\mathrm{m}^{3}\right)$.

\section{Cálculo da idade do lodo}

Durante os ensaios, diariamente era descartada ums quantidade de lodo para se manter a concentração de sólidos suspensos totais em, no máximo, 12 g.L-1 no reator biológico. No caso deste experimento, decidiu-se realizar o descarte direto do tanque de aeração. O cálculo da idade do lodo ou tempo de residência do lodo foi dado pela Equação 3.

Idade do Lodo $=\frac{\mathrm{V}}{\mathrm{Q}_{\mathrm{ex}}}$

Equação 3

Onde:

$\mathrm{V}$ : volume do reator biológico $\left(\mathrm{m}^{3}\right)$;

$\mathrm{Q}_{\mathrm{ex}}$ : vazão de descarte de lodo $\left(\mathrm{m}^{3} \cdot \mathrm{dia}^{-1}\right)$.

\section{Cálculo da eficiência de remoção}

Todos os dias foram calculadas as eficiências de remoção de matéria orgânica carbonácea e nitrogenada do sistema piloto. A eficiência de remoção foi calculada segundo a Equação 4

Eficiência de Remoção (\%) $=\frac{S_{0}-S}{S_{0}} \cdot 100 \%$ Equação 4

Onde:

S: DQO, DBO, concentração de NTK ou $\mathrm{N}^{-N H} 4^{+}$do efluente tratado;

$\mathrm{S}_{0}$ : DQO, DBO, concentração de NTK ou $\mathrm{N}^{-\mathrm{NH}} 4^{+}$do afluente bruto.

\section{Operação do sistema de ultrafiltração}

O sistema de ultrafiltração, assim como o tratamento biológico, era diariamente monitorado quanto ao fluxo de permeado por meio das membranas, da TMP e da necessidade de limpeza química. 


\section{Cálculo do fluxo}

O fluxo é definido como a vazão de permeado dividida pela área superficial total de membranas. O fluxo foi calculado por meio da Equação 5.

$J=\frac{Q_{p}}{A_{\text {sup }}}$

Equação 5

Onde:

J: fluxo $\left(\mathrm{L} \cdot \mathrm{m}^{-2} \cdot \mathrm{h}^{-1}\right)$;

$\mathrm{Q}_{\mathrm{p}}$ : vazão de permeado (L.h $\left.\mathrm{h}^{-1}\right)$;

$\mathrm{A}_{\text {sup }}$ : área do sistema de membranas $\left(\mathrm{m}^{2}\right)$.

\section{TMP}

É definida como a diferença entre a média da pressão de alimentação e do concentrado em relação à pressão de permeado. $\mathrm{O}$ modo de filtração tangencial de operação foi calculado pela Equação 6.

$\mathrm{TMP}=\left(\frac{\mathrm{P}_{\mathrm{A}}+\mathrm{P}_{\mathrm{C}}}{2}\right)-\mathrm{P}_{\mathrm{P}}$

\section{Equação 6 Resultados e discussão}

Onde:

TMP: pressão transmembrana (mbar);

$\mathrm{P}_{\mathrm{A}}$ : pressão da alimentação (mbar);

$\mathrm{P}_{\mathrm{C}}$ : pressão do concentrado (mbar);

$\mathrm{P}_{\mathrm{P}}$ : pressão do permeado (mbar).

\section{Determinação do fluxo crítico}

O fluxo crítico foi determinado a partir dos ensaios de longo tempo de operação. A variação da taxa da TMP no tempo ( $\triangle \mathrm{TMP} / \mathrm{dt})$ e cada fluxo testado foram relacionados num plano cartesiano, para se obter o fluxo crítico.
Para cada um dos ensaios, foi determinado o tempo sustentável $\left(\mathrm{t}_{\text {sust }}\right)$ de operação, ou seja, o tempo no qual a TMP apresenta súbito aumento, passando a apresentar perfil exponencial. Até esse período, não se observava a deposição (fouling) irreversível. Também foi ajustada uma curva de tendência exponencial (com o auxílio do software Excel $^{\circledR}$ ), de cada ensaio, para os períodos após o tsust.

Por meio das curvas ajustadas para cada ensaio de longo tempo, é possível calcular, por extrapolação, o tempo necessário para se atingir determinado valor de TMP. No caso, simulou-se o tempo necessário para se atingir a TMP máxima de 450 mbar (t450), que é indicativo de necessidade de limpeza química.

De posse dos tempos para atingir a TMP máxima, pode-se calcular a inclinação da reta, partindo da TMP inicial do ensaio até se atingir a TMP de 450 mbar. Essa inclinação é uma aproximação para a $\triangle \mathrm{TMP} / \mathrm{dt}$ e esta, quando relacionada com o respectivo fluxo testado, fornece indicação clara do fluxo crítico.

Para cada ensaio foi feita a extrapolação dos dados e o cálculo do tempo necessário para se realizar a limpeza química.

\section{Resultados do tratamento biológico}

As Tabelas 1 e 2 apresentam os resultados médios para o afluente bruto, efluente tratado e as condições do reator.

Os valores descritos na Tabela 2 representam os valores médios para todo o período do teste, porém a DQO afluente apresentou grandes oscilações e, na média, esteve em $2.505 \pm 520$ mg. $\mathrm{L}^{-1}$; em contrapartida, o MBR mostrou robustez, e a DQO efluente esteve, na média, em $59 \pm 27 \mathrm{mg} \cdot \mathrm{L}^{-1}$, exceto no período entre os dias 53 e 59, devido às constantes paradas para limpeza química e a consequente queda na $\operatorname{COV}\left(\mathrm{COV}=0,63 \mathrm{kgDQO} \cdot \mathrm{m}^{-3} \cdot \mathrm{d}^{-1}\right)-$ a DQO efluente

Tabela 1 - Condições operacionais durante os testes

\begin{tabular}{|c|c|c|c|c|}
\hline \multicolumn{5}{|c|}{ Condições operacionais } \\
\hline Parâmetro & Média & Desvio padrão & Máximo & Mínimo \\
\hline $\mathrm{A} / \mathrm{M}\left(\mathrm{kgDQO} \mathrm{kgSSV}^{-1} \cdot \mathrm{dia}^{-1}\right)$ & 0,170 & $\pm 0,040$ & 0,227 & 0,054 \\
\hline N/M (kgNTK.kgSSV-1.dia-1) & 0,018 & $\pm 0,018$ & 0,022 & 0,007 \\
\hline COV (kgDQO.m-3.dia-1) & 1,91 & $\pm 0,28$ & 2,17 & 0,63 \\
\hline CVN (kgNTK.m-3.dia-1) & 0,18 & $\pm 0,03$ & 0,26 & 0,07 \\
\hline Idade do lodo (dias) & 35 & $\pm 0,0$ & 35 & 35 \\
\hline Vazão de alimentação $\left(\right.$ L.h $\left.h^{-1}\right)$ & 59 & \pm 12 & 103 & 45 \\
\hline Temperatura $\left({ }^{\circ} \mathrm{C}\right)$ & 25 & $\pm 1,7$ & 28 & 20 \\
\hline Sólidos suspensos totais (mg.SST.L-1) & 11.407 & \pm 765 & 13.420 & 9.700 \\
\hline Sólidos suspensos voláteis (mg.SSV.L-1) & 10.641 & \pm 741 & 12.800 & 9.300 \\
\hline \multicolumn{5}{|l|}{ Oxigênio dissolvido (mg. $\mathrm{L}^{-1}$ ) } \\
\hline Anóxico & 0,1 & $\pm 0,1$ & 0,5 & 0,0 \\
\hline Aeróbio & 3,9 & $\pm 0,4$ & 5,0 & 3,1 \\
\hline
\end{tabular}

A/M: relação alimento/micro-organismo; N/M: relação nitrogênio/micro-organismos; COV: carga orgânica volumétrica; CVN: carga volumétrica nitrogenada. 
Tabela 2 - Características do afluente e efluente durante os testes

\begin{tabular}{|c|c|c|c|c|c|c|}
\hline \multirow{3}{*}{ Parâmetro } & \multicolumn{6}{|c|}{ Características do afluente e efluente } \\
\hline & \multicolumn{3}{|c|}{ Afluente } & \multicolumn{3}{|c|}{ Efluente } \\
\hline & Média & Máximo & Mínimo & Média & Máximo & Mínimo \\
\hline DQO (mg. $\left.\mathrm{L}^{-1}\right)$ & $2505 \pm 520$ & 3955 & 1540 & $59 \pm 27$ & 144 & 19 \\
\hline NTK (mg. $\left.L^{-1}\right)$ & $277 \pm 57$ & 461 & 156 & $3,7 \pm 2,4$ & 12 & 1,2 \\
\hline $\mathrm{N}-\mathrm{NH}_{4}{ }^{+}\left(\mathrm{mg} \cdot \mathrm{L}^{-1}\right)$ & $184 \pm 53$ & 268 & 52 & $0,6 \pm 0,2$ & 1,2 & 0,3 \\
\hline $\mathrm{N}-\mathrm{NO}_{2}^{-}\left(\mathrm{mg} \cdot \mathrm{L}^{-1}\right)$ & - & - & - & $0,10 \pm 0,20$ & 1,0 & 0,0 \\
\hline $\mathrm{N}-\mathrm{NO}_{3}^{-}\left(\mathrm{mg} \cdot \mathrm{L}^{-1}\right)$ & - & - & - & $17,8 \pm 11,3$ & 39,2 & 0,0 \\
\hline NT $\left(m g \cdot L^{-1}\right)$ & $277 \pm 53$ & 461 & 156 & $20,5 \pm 11,6$ & 44,3 & 3,3 \\
\hline SST $\left(m g \cdot L^{-1}\right)$ & - & - & - & $8 \pm 3$ & 18 & 3 \\
\hline \multirow[t]{2}{*}{ SSV $\left(m g \cdot L^{-1}\right)$} & - & - & - & $5 \pm 3$ & 15 & 1 \\
\hline & \multicolumn{6}{|c|}{ Eficiências de remoção (\%) } \\
\hline DQO & \multicolumn{6}{|c|}{$97,5 \pm 1,5$} \\
\hline NTK & \multicolumn{6}{|c|}{$98,6 \pm 0,8$} \\
\hline NT & \multicolumn{6}{|c|}{$92,1 \pm 4,2$} \\
\hline
\end{tabular}

DQO: demanda química de oxigênio; NTK: nitrogênio total Kjeldahl; NT: nitrogênio total; SST: sólidos suspensos totais; SSV: sólidos suspensos voláteis.

atingiu valores de $124 \pm 10 \mathrm{mg} \cdot \mathrm{L}^{-1}$. Após a regularização da COV, a DQO voltou a patamares normais. A Figura 2 apresenta o perfil de DQO para o período de testes.

A concentração de NTK no afluente apresentou uma média de $277 \pm 53 \mathrm{mg} \cdot \mathrm{L}^{-1}$, também bastante oscilante como a DQO, mas a atividade nitrificante desde o início da operação foi efetiva e a concentração de NTK no efluente tratado esteve em 3,7 \pm 2,4 mg. $\mathrm{L}^{-1}$. Nos primeiros dez dias, a concentração de NTK no afluente esteve em torno de 7,8 \pm 3,0 mg. $\mathrm{L}^{-1}$, provavelmente devido ao fato de o lodo não estar adaptado às condição de 35 dias de tempo de retenção celular impostas ao sistema.

O MBR apresentou boa atividade de nitrificação e isto foi comprovado pela baixa concentração de nitrogênio amoniacal no efluente tratado. A média de $\mathrm{N}-\mathrm{NH}_{4}+$ no efluente foi de 0,60 $\pm 0,24 \mathrm{mg} \cdot \mathrm{L}^{-1}$. A Figura 3 mostra o perfil de NT no afluente e efluente tratado.

A Figura 4 apresenta o perfil da remoção de DQO, NTK e NT, refletindo a remoção de material carbonáceo e nitrogenado (nas formas amoniacal, nitrito e nitrato). A variação na remoção de DQO entre os dias 53 a 59 reflete as variações na COV devido às paradas para realização de limpezas químicas, que foram diárias nesse período.

De forma geral os resultados alcançados são semelhantes aos econtrados em outros trabalhos com MBR, tal como os trabalhos de Zoh e Strenstrom (2002) e Chen et al. (2003), cujas eficiências de remoção de DQO e NTK são da mesma ordem de grandeza, ou seja, maior a 95\% no caso da DQO ou entre 85 e 90\% no caso do NTK. A remoção de NT, nesse caso, mostrou-se mais eficiente $(92,1 \%)$ do que o esperado, que seria $80 \%$, segundo a recirculação do reator aeróbio para o reator anóxico.



Figura 2 - Perfil de demanda química de oxigênio (DQO) afluente e efluente.

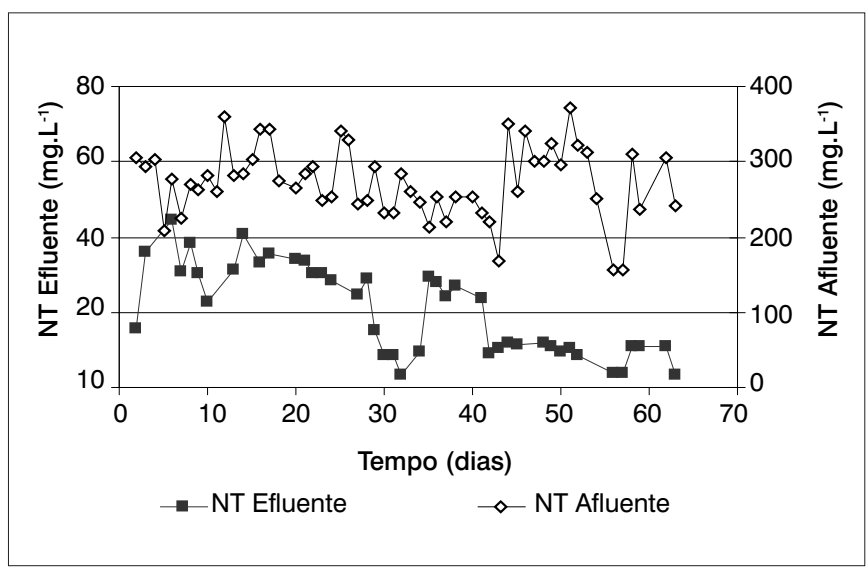

Figura 3 - Perfil de nitrogênio total (NT) afluente e efluente. 
É sabido que a recirculação entre o reator aeróbio e anóxico é fator fundamental na eficiência de remoção de NT, mas esta se manteve fixa em 4, pois não foi objetivo do trabalho estudar a influência da variação da recirculação de nitratos sobre as demandas da desnitrificação.

\section{Resultados da ultrafiltração}

A Figura 5 demonstra como a TMP se comportou durante cada ensaio para cada valor de fluxo bruto médio aplicado às membranas. No gráfico, fica evidente a influência do fluxo de filtração sobre a variação da TMP ao longo do tempo.

Como mencionado nos Métodos, para cada um dos ensaios foi determinado o tempo sustentável (tsust) de operação, ou seja, o tempo no qual a TMP apresenta súbito aumento, passando a apresentar perfil exponencial.

Os valores de cada tsust e t450 e de cada $\Delta \mathrm{TMP} / \mathrm{dt}$ são apresentados na Tabela 3. Os valores de tsust e t450 foram encontrados

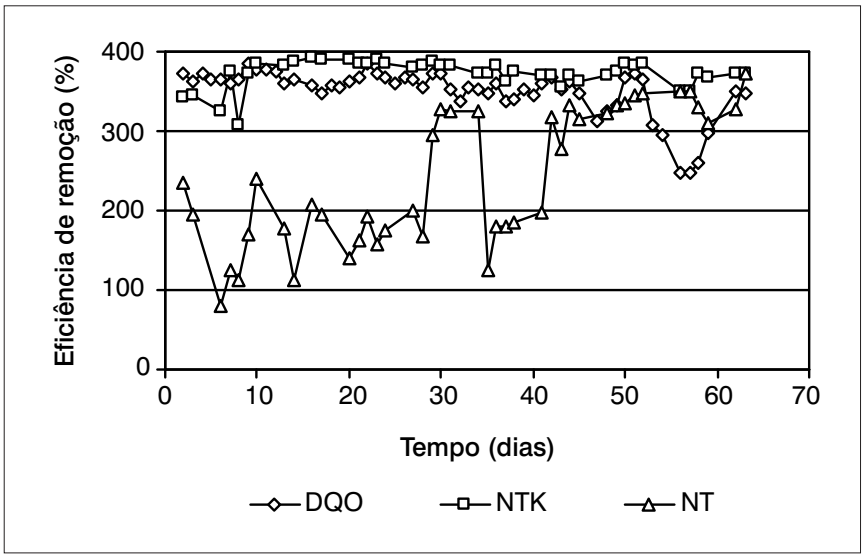

Figura 4 - Perfil de eficiência de remoção de demanda química de oxigênio (DQ0), nitrogênio total Kjeldahl (NTK) e nitrogênio total (NT) durante os testes. por meio de curvas ajustadas às coleções de dados em cada fluxo de operação - é evidente que quanto maior o fluxo, menor o tempo sustentável de operação.

A Figura 6 apresenta a relação da $\Delta \mathrm{TMP} / \mathrm{dt}$ com o respectivo fluxo em que ela ocorre. É possível observar que, a partir de $28 \mathrm{~L} \cdot \mathrm{m}^{-2} \cdot \mathrm{h}^{-1}$, há uma diferença considerável na taxa de variação da TMP, o que determina que, a partir desse fluxo, o processo se torna inviável do ponto de vista da ocorrência da deposição (fouling), pois a taxa de deposição se torna muito elevada, exigindo limpezas químicas em curto espaço de tempo.

É possível também relacionar o tsust com o fluxo aplicado. A Figura 7 demonstra a relação entre o tsust para cada fluxo testado. Pode-se notar que conforme o fluxo aumenta, o tempo para ocorrência da deposição irreversível diminui (tsust) e, a partir daquele momento, a TMP passa a variar exponencialmente no tempo, ou seja, quanto maior o fluxo mais rapidamente há necessidade de parada da unidade para a realização de limpezas químicas

Os resultados encontrados estão em concordância com a literatura, ou seja, sistemas MBR equipados com ultrafiltração de baixa TMP operam bem em fluxos na ordem de 15 a 20 L.m $\mathrm{m}^{-2} \cdot \mathrm{h}^{-1}$ (Guglielmi et al., 2007; Le Clech et al., 2006). A partir desses valores, a ocorrência da deposição irreversível, que exige paradas para limpeza química, passa ser muito frequente, compromentendo a capacidade de produção do sistema e inviabilizando o projeto.

Tabela 3 - Relação entre fluxo, $\mathrm{t}_{\text {sust }}, \mathrm{t}_{450}$ e $\Delta \mathrm{TMP} / \mathrm{dt}$

\begin{tabular}{lccc} 
Fluxo $\left(\mathrm{L} \cdot \mathrm{m}^{-2} \cdot \mathrm{h}^{-1}\right)$ & $\mathrm{t}_{\text {sust }}(\mathrm{h})$ & $\mathrm{t}_{450}(\mathrm{~h})$ & $\Delta \mathrm{TMP} / \mathrm{dt}\left(\mathrm{Pas}^{-1}\right)$ \\
\hline 25 & 97 & 176 & 0,100 \\
26 & 66 & 155 & 0,168 \\
28 & 55 & 123 & 0,182 \\
30 & 12 & 33 & 0,810 \\
37 & - & 14 & 0,595
\end{tabular}

$t$ : tempo sustentável; $t_{450}$ : tempo necessário para se atingir a TMP máxima de 450 mbar; STMP: diferencial da pressão transmembrana.

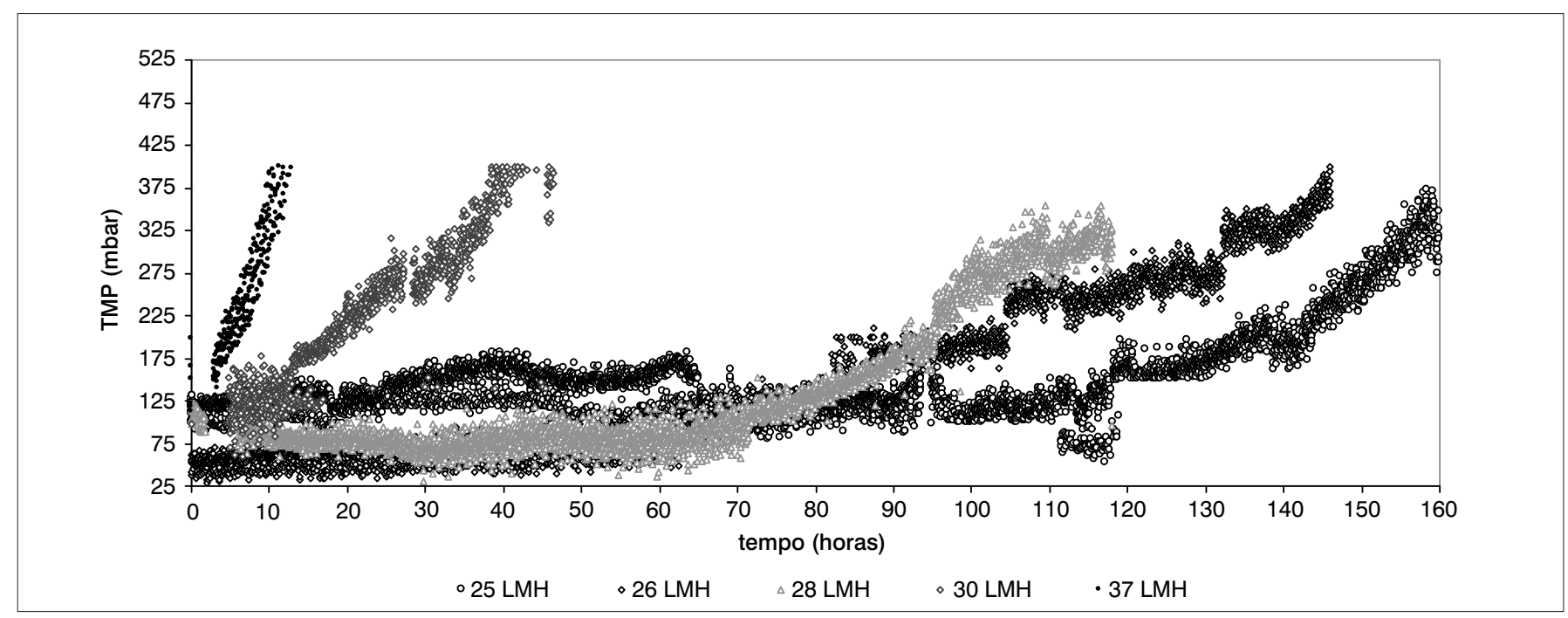

Figura 5 - Perfil da pressão transmembrana (TMP) em vários fluxos de filtração. 


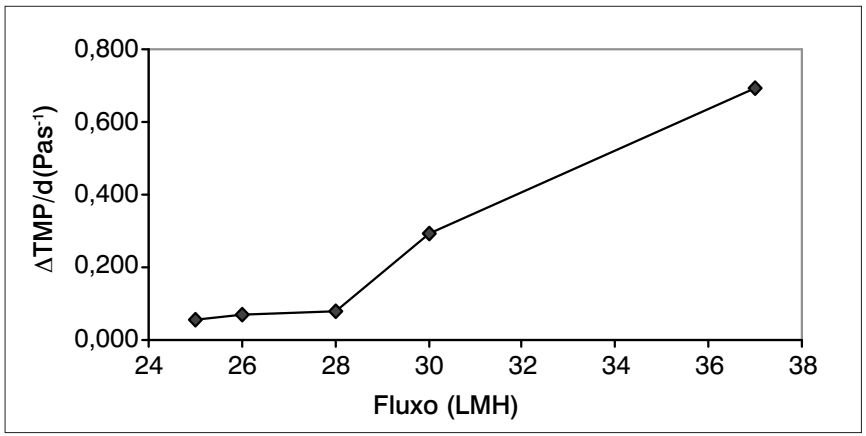

Figura 6 - Taxa de variação temporal da pressão transmembrana (TMP) em vários fluxos de filtração.

\section{Conclusões}

O sistema de separação sólido-líquido de ultrafiltração tubular de baixa pressão com inserção de ar, escolhido para filtração do efluente tratado, apresentou-se eficiente, produzindo um permeado clarificado com baixas concentrações de SST e SSV, respectivamente $8 \pm 3 \mathrm{mg} . \mathrm{L}^{-1}$ e $5 \pm 3 \mathrm{mg} . \mathrm{L}^{-1}$, operando com concentrações de $11,4 \pm 0,7$ g. $\mathrm{L}^{-1}$ e $10,6 \pm 0,7$ g. $\mathrm{L}^{-1}$, respectivamente, de SST e SSV no biorreator.



Figura 7 - Relação entre fluxo e tempo sustentável (tsust).

O fluxo crítico para o sistema de ultrafiltração tubular de baixa pressão é de $28 \mathrm{~L} \cdot \mathrm{m}^{-2} \cdot \mathrm{h}^{-1}$ quando operado nas condições de SST e SSV já mencionadas, com tempo de retenção celular de 35 dias.

Os valores médios de eficiência de remoção de DQO, NTK e NT foram de $97,5,98,6$ e 92,1\%, respectivamente, com o biorreator operando com COV 1,91 \pm 0,13 kgDQO.m-3.d-1 e CVN de 0,18 \pm 0,02 kgNTK.m ${ }^{-3}$. dia ${ }^{-1}$, com recirculação interna de nitratos de quatro vezes a vazão afluente. A DQO, o NTK e o NT, no efluente tratado, foram de $59 \pm 27 \mathrm{mgDQO} . \mathrm{L}^{-1}$, 3,7 $\pm 2,4$ mgNTK.L $L^{-1}$ e 20,5 $\pm 11,9$ mgNT. $L^{-1}$, respectivamente.

\section{Referências}

APHA, AWWA, WPCF. Standard methods for the examination of water and wastewater. 21. ed. Washington, DC, 2005.

BACHIM, P.; AIMAR, P.; FIELD, R.W. Critical and sustainable fluxes: theory, experiments and applications. Journal of Membrane Science, v. 286, p. 42-69, 2006

CAGATAYHAN, B.E. Comparison of recirculation configurations for biological nutrient removal in a membrane bioreactor. Water Research, v. 42, p. 1651-1663, 2008

CHEN, T. K. et al. High-strength nitrogen removal of epto-eletronic industrial wastewater in membrane bioreactor - a pilot study. Water Science and Technology, v. 48, p. 1191-1198, 2003

ERSU, C.B. et al. Comparison of recirculation configurations for nutrient removal in a membrane bioreactor. Water Research, v. 42, p. 1651-1663, 2008.

GUGLIELMI, G. et al. Sub-critical fouling in a membrane bioreactor for municipal wastewater treatment: Experimental investigation and mathematical modeling. Water Research, v. 41, p. 3903-3914, 2007

JUDD, S. The MBR Book: principles and applications of membrane bioreactors in water and wastewater treatment. London: Elsevier, 2006. 325p
LE CLECH, P.; CHEN, V.; FANE, A.G. Fouling in membrane bioreactors used in wastewater treatment. Journal of Membrane Science, v. 284, p. 17-53, 2006

METCALF \& EDDY, INC., Wastewater engineering: treatment and reuse. 4. ed. New Delhi: McGraw Hill, 2003.

MENG, F. et al. Recent advances in membrane bioreactors (MBRs): Membrane fouling and membrane material. Water Research, v. 43, p. 1489-1512, 2009

NYWENING, J.P.; ZHOU, H. Influence of filtration conditions on membrane fouling and scouring aeration effectiveness in submerged membrane bioreactors to treat municipal wastewater, Water Research, v. 43, p. 3548-3558, 2009

WATER ENVIRONMENT FEDERATION (WEF). Biological Nutrient Removal Operation in Wastewater Treatment Plant: Manual of Practice No. 29. New York. Mc Graw Hill, 2005

YUAN, D. et al. Study of a membrane bioreactor with glass fiber flat grille modules and the modules' optimization based on the local critical flux theory. Water Research, v. 44, p. 997-1005, 2010.

ZOH, K.D.; STENSTROM, M.K. Application of a membrane bioreactor for treating explosives process wastewater. Water Research, v. 36, p. 10181024, 2002. 